\title{
Influence of capillarity on a simple harmonic oscillating water table: Sand column experiments and modeling
}

\author{
Nick Cartwright \\ School of Engineering, Griffith University, Gold Coast, Queensland, Australia \\ Peter Nielsen \\ Division of Civil Engineering, University of Queensland, St. Lucia, Queensland, Australia
}

Pierre Perrochet

Centre of Hydrogeology, University of Neuchâtel, Neuchâtel, Switzerland

Received 7 February 2005; revised 24 May 2005; accepted 1 June 2005; published 19 August 2005.

[1] Comprehensive measurements of the water table response to simple harmonic forcing at the base of a sand column are presented and discussed. In similar experiments, Nielsen and Perrochet (2000) observed that fluctuations in the total moisture were both damped and lagged relative to the water table fluctuations. As a result, the concept of a complex effective porosity was proposed as a convenient means to account for the damping and phase lag through its magnitude and argument, respectively. The complex effective porosity then enables simple analytical solutions for the water table (and total moisture) dynamics including hysteresis. In this paper, these previous experiments are extended to cover a wider range of oscillation frequencies and are conducted for three well-sorted materials with median grain diameters of $0.082,0.2$, and $0.78 \mathrm{~mm}$, respectively. In agreement with existing theory, the influence of the capillary fringe is shown to increase with the oscillation frequency. However, the complex effective porosity model corresponding to the classical Green and Ampt (1911) capillary tube approximations is shown to be inadequate when compared to the data. These limitations are overcome by the provision of an empirical, frequency-dependent complex effective porosity model fit to the data. Using measured moisture retention parameters, numerical simulation of the data solving a nonhysteretic van Genuchten-Richards' equation type model is unable to replicate the observations. Existing results of a hysteretic numerical model are shown to be in good agreement with the extended database.

Citation: Cartwright, N., P. Nielsen, and P. Perrochet (2005), Influence of capillarity on a simple harmonic oscillating water table: Sand column experiments and modeling, Water Resour. Res., 41, W08416, doi:10.1029/2005WR004023.

\section{Introduction}

[2] This experimental study of the influence of the capillary fringe on simple harmonic water table oscillations has been motivated by a wider research investigation into the dynamic coupling of the ocean with coastal aquifers. Water table fluctuations in coastal aquifers are important for the exchange processes and mixing that occurs between the ocean and groundwater. This will have implications for issues such saltwater intrusion and contaminant transport and degradation.

[3] Another important issue relating to the coupling of the near-shore ocean with beach aquifers is the mobility of sediments on the beach face [e.g., Elfrink and Baldock, 2002]. This in turn has wider implications for coastal erosion in general.

[4] The influence of the capillary fringe on water table oscillations has received much attention in the literature from experimental [e.g., Lehman et al., 1998; Nielsen and

Copyright 2005 by the American Geophysical Union. 0043-1397/05/2005WR004023
Perrochet, 2000; Stauffer and Kinzelbach, 2001], theoretical [e.g., Parlange and Brutsaert, 1987; Barry et al., 1996; Nielsen and Perrochet, 2000; Li et al., 2000] and numerical [e.g., Li et al., 1997; Stauffer and Kinzelbach, 2001; Werner and Lockington, 2003] perspectives.

[5] Although it has been shown that, upon consideration of hysteresis effects, numerical solution of Richards' [1931] unsaturated flow equation can accurately reproduce experimental observations [e.g., Stauffer and Kinzelbach, 2001; Werner and Lockington, 2003], the computational effort required for such simulations is high and simulation time can be greater than the actual experimental period. To simplify the problem, efforts to incorporate the effects of capillarity into analytical developments have adopted the classical Green and Ampt [1911] approximations of the fringe [e.g., Parlange and Brutsaert, 1987; Barry et al., 1996; Li et al., 2000].

[6] Green and Ampt [1911] approximated the nature of the fringe by assuming that it is completely saturated to its top, above which zero moisture exists. At the top of the fringe the suction head is constant at $-H_{\psi}$. Also the hydraulic conductivity within the fringe behaves according 
to the constant saturated value as defined for below the water table. This approximation however is inherently nonhysteretic. Through experimental observations of simple harmonic water table oscillations in a sand column, this paper highlights limitations in both the Green and Ampt [1911] approximation and nonhysteretic numerical solvers.

[7] Nielsen and Perrochet [2000] introduced the concept of a dynamic complex effective porosity $n_{\omega}$ in order to account implicitly for hysteresis effects on an oscillating water table. The complex nature of $n_{\omega}$ was required to account for the observation that fluctuations in the equivalent saturated height of the total moisture $\mathrm{d} h_{\text {tot }}$ were both damped and lagged relative to those in water table $\mathrm{d} h$.

[8] It is noted that such a parameterization of the influence of the capillary fringe into a dynamic storage coefficient does not provide information on the dynamics of the moisture distribution above the water table. It does however provide a simple, and computationally efficient way to account for its influence on the nature of water table fluctuations and is easily applied to existing dispersion relation theories to describe water table attenuation [e.g., Nielsen and Turner, 2000; Cartwright et al., 2003].

[9] From their experiments over the range of oscillation periods, $14 \mathrm{~min}<T<6.5$ hours, Nielsen and Perrochet [2000] concluded that the complex effective porosity was a constant for the sand they tested. In this paper, further sand column experiments are conducted over a wider range of oscillation frequencies and for two additional porous materials with the results indicating a strong dependence of capillarity effects $\left(n_{\omega}\right)$ on oscillation frequency.

[10] In section 2 of this paper the concept of a complex effective porosity is briefly described for reference. Section 3 describes the experimental and data analysis procedures followed by a discussion of the results in section 4 . The limitations of the nonhysteretic Green and Ampt [1911] and Richards [1931] equation type models when applied to the data are described in sections 5 and 6 respectively. The successful comparison of hysteretic Richards [1931] equation predictions with the data is provided in section 7 , and conclusions are drawn in section 8 .

\section{Dynamic Complex Effective Porosity Concept}

[11] The fundamental definition of the complex effective porosity is [Nielsen and Perrochet, 2000]

$$
n \frac{\mathrm{d} h_{t o t}}{\mathrm{~d} t}=n_{\omega} \frac{\mathrm{d} h}{\mathrm{~d} t}
$$

where $n_{\omega}$ is the complex effective porosity and $n$ is the drainable porosity.

[12] Nielsen and Perrochet [2000] observed that fluctuations in the equivalent saturated height of the total moisture $h_{\text {tot }}$ were both damped and lagged relative to those in the water table $h$ (see their Figure 4). Therefore the complex nature of $n_{\omega}$ was adopted to account for the damping through its magnitude $\left|n_{\omega}\right|$ and the phase lag through its argument $\operatorname{Arg}\left\{n_{\omega}\right\}$. Note that we have elected to use the subscript $\omega$ as opposed to Nielsen and Perrochet's [2000] $n_{d}$ to denote the dependence of the complex effective porosity

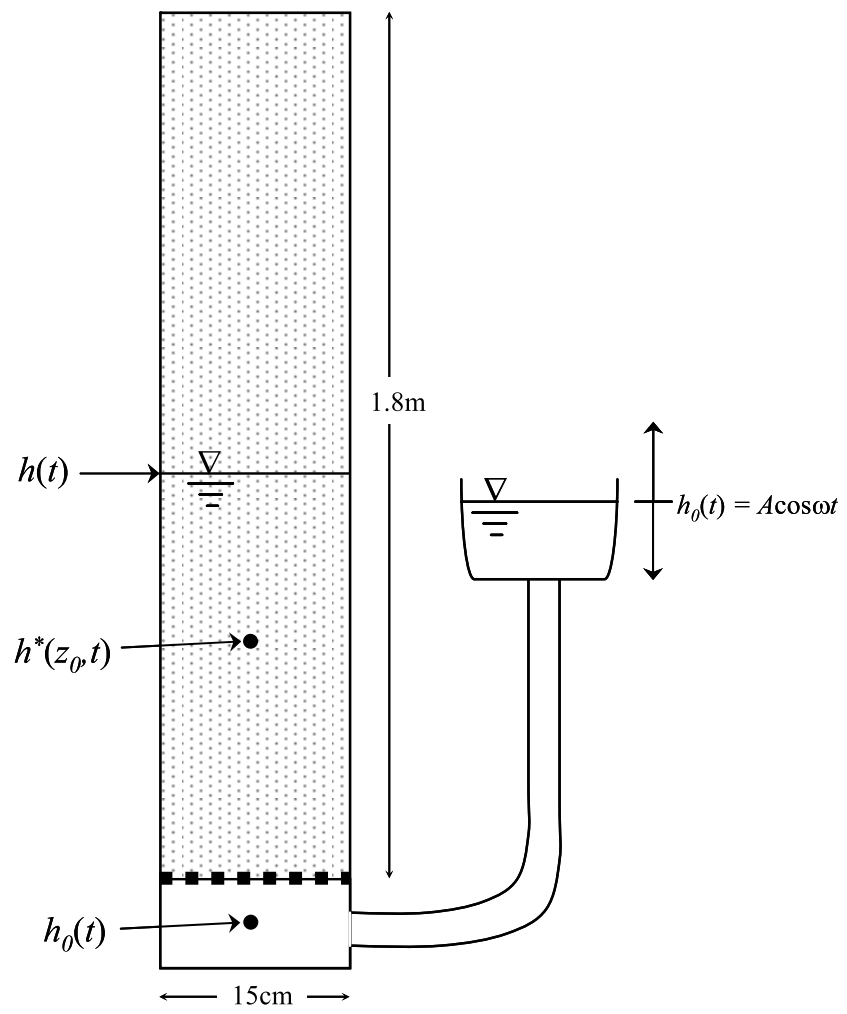

Figure 1. Schematic of the sand column.

on the oscillation frequency $\omega$. This will be clearly evidenced in section 4 .

\section{Experimental Procedure}

[13] The sand column shown in Figure 1 is the same as described in detail by Nielsen and Perrochet [2000]. The pore pressure in the saturated zone and the driving head level were measured using pressure transducers and the water table elevation was inferred using the following equation [Nielsen and Perrochet, 2000],

$$
h(t)=\frac{z_{0} h_{0}(t)}{z_{0}+h_{0}(t)-h^{*}\left(z_{0}, t\right)}
$$

where $h_{0}$ is the driving head level, $z_{0}$ is some elevation always below the water table and $h^{*}\left(z_{0}, t\right)$ is the piezometric head measured at the elevation $z_{0}$ (see Figure 1).

[14] In an extension of the experiments conducted by Nielsen and Perrochet [2000], the range of forcing frequencies was extended down to a period of $10 \mathrm{~s}$ which is the typical period of wind wave fluctuations. Also covered were periods of the order of hundreds of seconds, corresponding to oceanic long waves that occur naturally in the surf zone.

\subsection{Sand Parameters}

[15] The beaches in the general study region of south east Queensland and northern New South Wales in Australia consist of quartz dominated, well-sorted sands. The present experiments used three well-sorted sands of varying grain sizes in order to have a range of capillary fringe heights. Two were natural sands $\left(d_{50}=0.78 \mathrm{~mm}\right.$ and $0.2 \mathrm{~mm}$ respectively) with the $d_{50}=0.2 \mathrm{~mm}$ sand being locally 
mined from the dune system on nearby North Stradbroke Island. The third "soil" was fine glass beads $\left(d_{50}=\right.$ $0.082 \mathrm{~mm}$ ).

[16] The sieve curves and first drying moisture retention curves are shown in Figures 2 and 3, respectively. The curves indicate the well-sorted nature of the sediments and the corresponding sharp transitions from saturated to residual moisture contents.

[17] Throughout the following analyses the capillary fringe is represented by an equivalent steady saturated height $H_{\psi}$ computed from the measured first drying curves using,

$$
H_{\psi}=\int_{h}^{\infty} \frac{\theta-\theta_{r}}{\theta_{s}-\theta_{r}} d \psi
$$

where $\theta, \theta_{s}$ and $\theta_{r}$ are the local, saturated and residual moisture contents respectively and $\psi$ is the suction head.

[18] This is considered a reasonable representation for the three well-sorted materials considered here due to their fairly rapid transitions from saturated to residual moisture contents (see Figure 3). For more poorly sorted materials where this transition is much more gradual, for example those with high clay contents, such a description may not necessarily be valid. Quantification of just how applicable this representation may be for such soils is considered beyond the scope of the present investigation into the behavior of well-sorted soils. It is noted however, that such investigation is warranted for future experiments.

[19] In each case the material was packed in the column by allowing a thin layer to settle through water to avoid air encapsulation and then thoroughly mixing with adjacent layers to remove any layering due to preferential settling. The procedure was repeated until the column was full of sediment. The saturated hydraulic conductivity was then determined in situ using a falling head test on the column as whole, the results from which therefore represent a column-

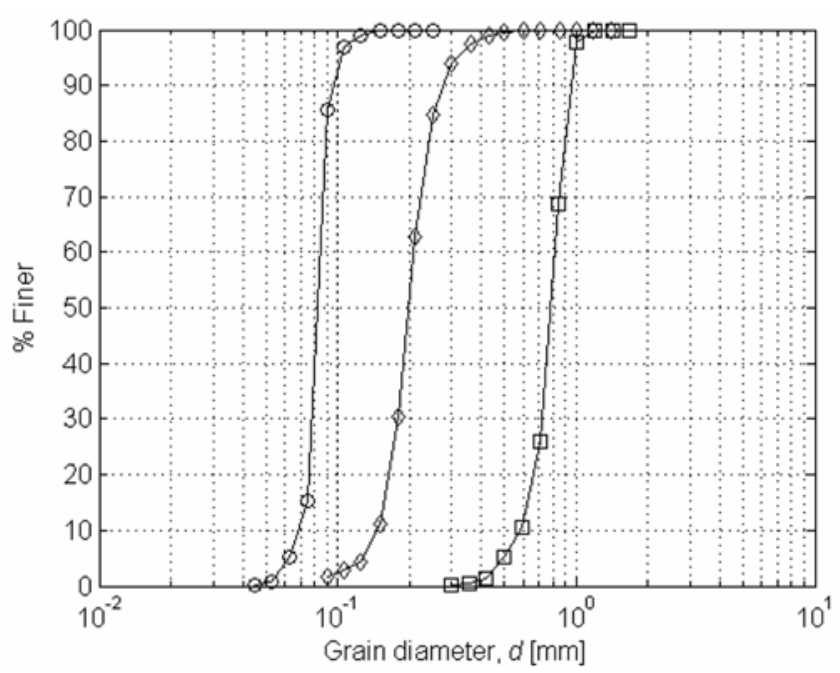

Figure 2. Sieve curves for the three test materials. The symbols denote $d_{50}=0.082 \mathrm{~mm}$ (circles), $d_{50}=0.2 \mathrm{~mm}$ (diamonds), and $d_{50}=0.78 \mathrm{~mm}$ (squares).
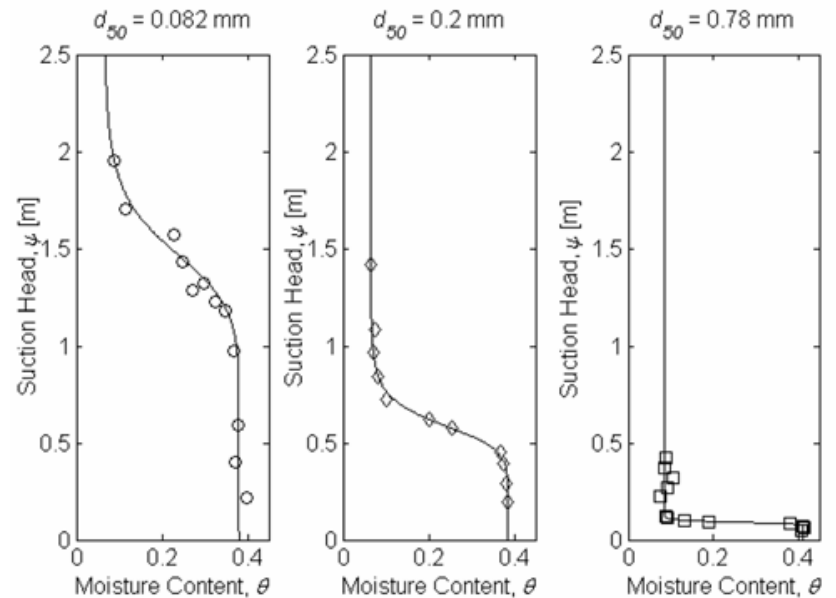

Figure 3. Measured first drying curves for the three test materials. Solid lines denote the best fit van Genuchten [1980] moisture-pressure relationships.

averaged value. The hydraulic properties of each material are summarized in Table 1.

\subsection{Experimental Determination of the Complex Effective Porosity}

[20] The experimental complex effective porosity was estimated based on the assumption of a linear response of the water table. This technique was validated by Nielsen and Perrochet [2000] against values they derived from the definition (1) via direct measurements of the moisture distribution in the column using the gamma radiation absorption technique. The function describing the linear response of the water table to simple harmonic forcing in the column is [Nielsen and Perrochet, 2000]

$$
F(\omega)=\frac{\eta(t)}{\eta_{0}(t)}=|F| e^{-i \phi}=\frac{1}{1+i \omega \frac{n_{\omega} D}{K}}
$$

where $|F|=|\eta| /\left|\eta_{0}\right|$ and $\phi$ are respectively the measured amplitude ratio and phase lag between the water table $(\eta(t))$ and the driving head $\left(\eta_{0}(t)\right), i$ is the imaginary number, $\omega$ is the angular frequency, $D$ is the mean aquifer thickness, $K$ is the saturated hydraulic conductivity and $n_{\omega}$ is the complex effective porosity. Amplitudes and phases are extracted from measured time series of $\eta(t)$ and $\eta_{0}(t)$ via harmonic analysis and equation (4) is then solved for $n_{\omega}$.

\section{Experimental Results}

[21] The experimental database is summarized in Table 2. A total of 63 tests were conducted using the three different materials. The experimentally determined values of $n_{\omega}$ are shown in Figure 4. The data show a clear trend adhered to by all three materials in that $n_{\omega}$ is strongly dependent upon $n \omega H_{\psi} / K$ in agreement with previous analytical [Barry et al., 1996] and numerical [Li et al., 1997] modeling work. That is, at low oscillation frequencies $\left(n \omega H_{\psi} / K \rightarrow 0\right)$ the influence of capillarity has little effect $\left(n_{\omega} \rightarrow n\right)$ and at higher frequencies $\left(n \omega H_{\psi} / K \rightarrow \infty\right)$ the influence of capillarity is greatly increased $\left(n_{\omega} \ll n\right)$. 
Table 1. Basic Parameters of the Three Test Materials

\begin{tabular}{clcccccc}
\hline Test Numbers & Material & $\begin{array}{c}d_{50}, \\
\mathrm{~mm}\end{array}$ & \multicolumn{1}{c}{$d_{90} / d_{10}$} & $K, \mathrm{~m} \mathrm{~s}^{-1}$ & $\begin{array}{c}\theta_{s}, \\
\mathrm{~cm}^{3} \mathrm{~cm}^{-3}\end{array}$ & $\begin{array}{c}\theta_{r}, \\
\mathrm{~cm}^{3} \mathrm{~cm}^{-3}\end{array}$ & $H_{\psi}, \mathrm{m}$ \\
\hline $1-21$ & glass beads & 0.082 & 1.4 & $2.8 \times 10^{-5}$ & 0.38 & 0.06 & 1.5 \\
$22-27$ & fine sand & 0.20 & 1.83 & $1.47 \times 10^{-4}$ & 0.38 & 0.09 & 0.6 \\
$28-55$ & fine sand & 0.20 & 1.83 & $1.0 \times 10^{-4}$ & 0.38 & 0.09 & 0.6 \\
$56-63$ & coarse sand & 0.78 & 1.6 & $2.5 \times 10^{-3}$ & 0.41 & 0.08 & 0.085 \\
\hline
\end{tabular}

[22] Plotted on a log-log scale, the asymptotic nature of the data as $n \omega H_{\psi} / K \rightarrow \infty$ indicates a power relationship between $n_{\omega}$ and $n \omega H_{\psi} / K$. The curves shown in Figure 4 are an empirical curve fit to the data with the following form,

$$
n_{\omega}=\frac{n}{1+2.5\left(i \frac{n \omega H_{\psi}}{K}\right)^{2 / 3}}
$$

That is, for large $n \omega H_{\psi} / K$, the damping of the total moisture relative to the water table $\left|n_{\omega}\right|$ goes as $\left(n \omega H_{\psi} / K\right)^{-2 / 3}$ and the phase lag $-\operatorname{Arg}\left\{n_{\omega}\right\}$ asymptotes to $\approx-\pi / 3$. The form of this equation will be shown to be analogous to, but markedly different from that derived for the Green and Ampt [1911] approximation in section 5 .

\subsection{Nonlinear Effects}

[23] In the experimental determination of the complex effective porosity outlined in section 3.2, a linear response of the water table is assumed. That is the governing flow

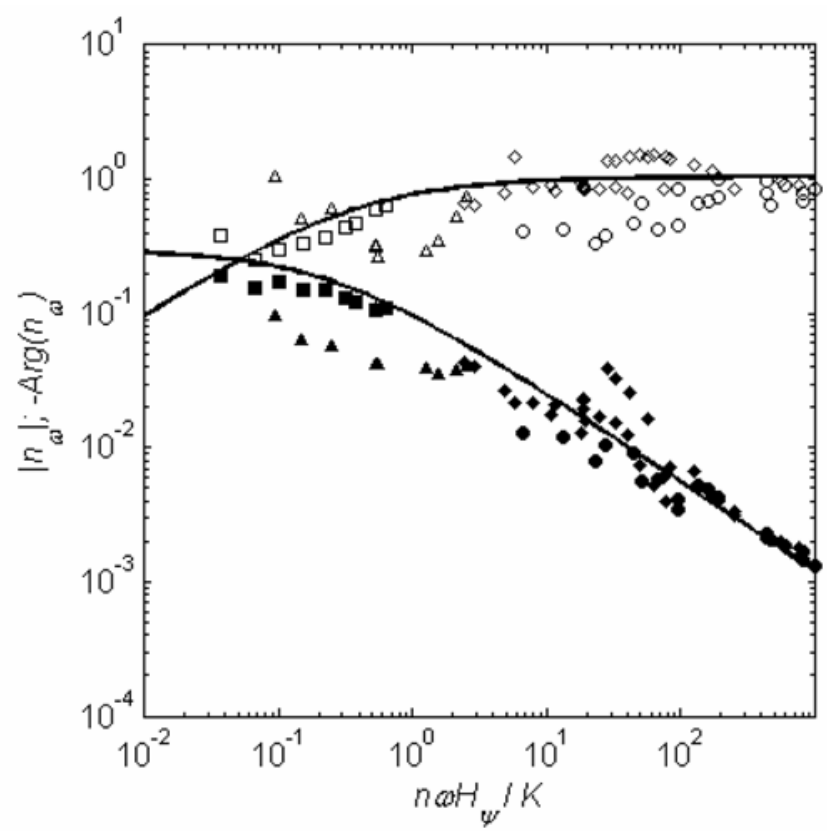

Figure 4. Effective porosities determined experimentally for three different sediments: $d_{50}=0.78 \mathrm{~mm}$ sand (squares), $d_{50}=0.2 \mathrm{~mm}$ sand (diamonds), and $d_{50}=0.082 \mathrm{~mm}$ glass beads (circles). Solid symbols denote $\left|n_{\omega}\right|$, and open symbols denote $-\operatorname{Arg}\left\{n_{\omega}\right\}$. The triangles denote data from Nielsen and Perrochet [2000] for $d_{50}=0.2 \mathrm{~mm}$ sand. The curve is an empirical fit to the data, (equation 5) (solid lines). equation is linearized under the assumption of a small amplitude oscillation, i.e., $|\eta| \ll D$. To investigate the influence, if any, of nonlinear (finite amplitude) effects, several experiments were conducted in which all other parameters were held constant and the amplitude increased incrementally for each test. The results of these tests are presented in Figure 5.

[24] The data indicate a weak dependence of $n_{\omega}$ on $|\eta| / D$ with both $\left|n_{\omega}\right|$ and $-\operatorname{Arg}\left\{n_{\omega}\right\}$ increasing slightly with increasing $|\eta| / D$, implying that capillarity effects are slightly more important for smaller oscillation amplitudes. However, the overall increase in $n_{\omega}$ for the range of tests conducted falls within the range of experimental scatter shown in Figure 4, indicating that any nonlinear effects are secondary to the dominant frequency dependence of capillarity effects.

\subsection{Influence of Proximity to the Sand Surface}

[25] The proximity of the surface will influence moisture exchange through a reduction in the aquifer storage coefficient [e.g., Duke, 1972; Gillham, 1984; Nachabe, 2002]. Cartwright et al. [2004] present data from the same sand column where all other parameters were kept constant and the sand surface lowered incrementally for each test, thereby truncating the capillary fringe. The results showed a significant reduction in $\left|n_{\omega}\right|$ of up to three orders of magnitude (relative to the truncation free value) as the fringe became increasingly truncated (see their Figure 8). This is in agreement with previous findings of a reduced specific yield under a shallow, steadily draining water table [e.g., Duke, 1972; Gillham, 1984; Nachabe, 2002].
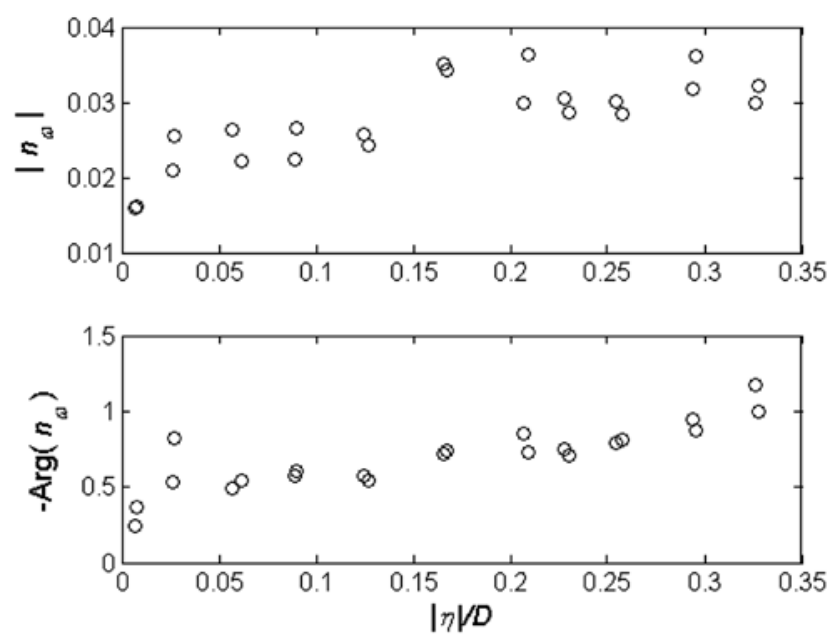

Figure 5. The influence of finite amplitude on the experimental complex effective porosity. 
Table 2. Summary of Experimental Results ${ }^{\mathrm{a}}$

\begin{tabular}{|c|c|c|c|c|c|c|c|}
\hline Test Number & $T, \mathrm{~s}$ & $D, \mathrm{~m}$ & $\left|\eta_{0}\right|, \mathrm{m}$ & $|\eta|, \mathrm{m}$ & $\phi_{w t}, \mathrm{rad}$ & $\Re\left\{n_{\omega}\right\}$ & $\Im\left\{n_{\omega}\right\}$ \\
\hline 1 & 1785 & 0.554 & 0.174 & 0.142 & 0.151 & 0.0027 & -0.0031 \\
\hline 2 & 1785 & 0.552 & 0.169 & 0.150 & 0.192 & 0.0031 & -0.0015 \\
\hline 3 & 2460 & 0.549 & 0.173 & 0.150 & 0.230 & 0.0052 & -0.0024 \\
\hline 4 & 1230 & 0.549 & 0.174 & 0.126 & 0.303 & 0.0041 & -0.0031 \\
\hline 5 & 870 & 0.569 & 0.174 & 0.116 & 0.318 & 0.0032 & -0.0029 \\
\hline 6 & 3780 & 0.569 & 0.171 & 0.147 & 0.234 & 0.0080 & -0.0040 \\
\hline 7 & 7380 & 0.569 & 0.169 & 0.160 & 0.124 & 0.0075 & -0.0026 \\
\hline 8 & 1050 & 0.570 & 0.174 & 0.121 & 0.321 & 0.0037 & -0.0030 \\
\hline 9 & 6150 & 0.569 & 0.166 & 0.151 & 0.187 & 0.0099 & -0.0040 \\
\hline 10 & 25200 & 0.572 & 0.168 & 0.163 & 0.058 & 0.0117 & -0.0051 \\
\hline 11 & 12600 & 0.570 & 0.166 & 0.157 & 0.107 & 0.0111 & -0.0050 \\
\hline 12 & 390 & 0.510 & 0.172 & 0.107 & 0.237 & 0.0013 & -0.0019 \\
\hline 13 & 283 & 0.516 & 0.169 & 0.102 & 0.283 & 0.0011 & -0.0014 \\
\hline 14 & 900 & 0.515 & 0.173 & 0.117 & 0.196 & 0.0022 & -0.0035 \\
\hline 15 & 170 & 0.514 & 0.175 & 0.099 & 0.339 & 0.0009 & -0.0010 \\
\hline 16 & 210 & 0.516 & 0.175 & 0.101 & 0.424 & 0.0013 & -0.0010 \\
\hline 17 & 140 & 0.515 & 0.175 & 0.094 & 0.521 & 0.0011 & -0.0007 \\
\hline 18 & 385 & 0.510 & 0.171 & 0.113 & 0.298 & 0.0015 & -0.0015 \\
\hline 19 & 210 & 0.511 & 0.169 & 0.101 & 0.341 & 0.0010 & -0.0010 \\
\hline 20 & 360 & 0.511 & 0.174 & 0.117 & 0.365 & 0.0017 & -0.0012 \\
\hline 21 & 3300 & 0.508 & 0.173 & 0.153 & 0.137 & 0.0045 & -0.0034 \\
\hline 22 & 137 & 0.602 & 0.175 & 0.088 & 0.384 & 0.0040 & -0.0045 \\
\hline 23 & 524 & 0.599 & 0.171 & 0.103 & 0.317 & 0.0106 & -0.0118 \\
\hline 24 & 890 & 0.594 & 0.172 & 0.115 & 0.275 & 0.0143 & -0.0155 \\
\hline 25 & 547 & 0.621 & 0.173 & 0.086 & 0.366 & 0.0148 & -0.0181 \\
\hline 26 & 547 & 0.745 & 0.173 & 0.081 & 0.414 & 0.0148 & -0.0164 \\
\hline 27 & 552 & 0.495 & 0.171 & 0.104 & 0.276 & 0.0117 & -0.0152 \\
\hline 28 & 10.3 & 0.530 & 0.151 & 0.045 & 10.918 & 0.0010 & 0.0007 \\
\hline 29 & 14.4 & 0.553 & 0.153 & 0.042 & 10.611 & 0.0015 & 0.0005 \\
\hline 30 & 27 & 0.534 & 0.148 & 0.053 & 10.051 & 0.0020 & -0.0003 \\
\hline 31 & 60.5 & 0.557 & 0.146 & 0.055 & 0.473 & 0.0021 & -0.0024 \\
\hline 32 & 12.6 & 0.551 & 0.154 & 0.046 & 10.809 & 0.0012 & 0.0007 \\
\hline 33 & 61 & 0.538 & 0.145 & 0.054 & 0.485 & 0.0023 & -0.0025 \\
\hline 34 & 20.1 & 0.551 & 0.152 & 0.048 & 10.322 & 0.0018 & 0.0001 \\
\hline 35 & 87.9 & 0.536 & 0.144 & 0.056 & 0.269 & 0.0018 & -0.0039 \\
\hline 36 & 269.8 & 0.542 & 0.144 & 0.047 & 0.065 & 0.0016 & -0.0163 \\
\hline 37 & 121.3 & 0.561 & 0.145 & 0.050 & 0.179 & 0.0018 & -0.0064 \\
\hline 38 & 309.3 & 0.551 & 0.146 & 0.080 & 0.033 & 0.0005 & -0.0074 \\
\hline 39 & 195.5 & 0.543 & 0.148 & 0.087 & 0.032 & 0.0003 & -0.0040 \\
\hline 40 & 178.9 & 0.530 & 0.145 & 0.062 & 0.079 & 0.0010 & -0.0072 \\
\hline 41 & 468.5 & 0.533 & 0.142 & 0.043 & 0.149 & 0.0069 & -0.0317 \\
\hline 42 & 240.1 & 0.541 & 0.142 & 0.082 & 0.024 & 0.0003 & -0.0052 \\
\hline 43 & 10.4 & 0.539 & 0.152 & 0.041 & 10.773 & 0.0011 & 0.0005 \\
\hline 44 & 530 & 0.543 & 0.142 & 0.041 & 0.146 & 0.0078 & -0.0377 \\
\hline 45 & 359.4 & 0.542 & 0.143 & 0.042 & 0.061 & 0.0022 & -0.0253 \\
\hline 46 & 3120 & 0.756 & 0.169 & 0.128 & 0.215 & 0.0185 & -0.0190 \\
\hline 47 & 1935 & 0.753 & 0.168 & 0.116 & 0.242 & 0.0142 & -0.0166 \\
\hline 48 & 840 & 0.759 & 0.169 & 0.103 & 0.271 & 0.0077 & -0.0102 \\
\hline 49 & 1410 & 0.754 & 0.168 & 0.111 & 0.251 & 0.0112 & -0.0139 \\
\hline 50 & 2610 & 0.760 & 0.169 & 0.121 & 0.025 & 0.0019 & -0.0217 \\
\hline 51 & 5160 & 0.756 & 0.168 & 0.134 & 0.234 & 0.0316 & -0.0238 \\
\hline 52 & 6120 & 0.759 & 0.169 & 0.137 & 0.216 & 0.0340 & -0.0263 \\
\hline 53 & 380 & 0.749 & 0.163 & 0.069 & 0.479 & 0.0088 & -0.0088 \\
\hline 54 & 466 & 0.749 & 0.165 & 0.069 & 0.435 & 0.0100 & -0.0116 \\
\hline 55 & 600 & 0.754 & 0.164 & 0.074 & 0.416 & 0.0113 & -0.0130 \\
\hline 56 & 1320 & 0.589 & 0.173 & 0.163 & 0.158 & 0.1482 & -0.0380 \\
\hline 57 & 2370 & 0.587 & 0.172 & 0.164 & 0.105 & 0.1765 & -0.0699 \\
\hline 58 & 386.3 & 0.589 & 0.163 & 0.123 & 0.423 & 0.1420 & -0.0546 \\
\hline 59 & 163 & 0.599 & 0.155 & 0.088 & 0.485 & 0.0888 & -0.0603 \\
\hline 60 & 137 & 0.600 & 0.151 & 0.077 & 0.508 & 0.0867 & -0.0648 \\
\hline 61 & 573 & 0.601 & 0.168 & 0.141 & 0.321 & 0.1425 & -0.0494 \\
\hline 62 & 278 & 0.601 & 0.159 & 0.109 & 0.463 & 0.1198 & -0.0558 \\
\hline 63 & 234 & 0.597 & 0.158 & 0.104 & 0.479 & 0.1093 & -0.0546 \\
\hline
\end{tabular}

${ }^{\mathrm{a}} T$ is the oscillation period, $D$ is the mean driving head level, $\left|\eta_{0}\right|$ and $|\eta|$ are the driving head and water table amplitudes, $\phi_{w t}$ is the water table phase lag, and $\Re\left\{n_{\omega}\right\}$ and $\Im\left\{n_{\omega}\right\}$ are the real and imaginary parts, respectively, of the complex effective porosity.

[26] In the present experiments however, the setup was such that truncation effects had no influence on the results. Therefore the empirical complex effective porosity model, equation (5), is only valid where the water table is deep enough such that truncation effects are negligible. As a guide the experiments of Cartwright et al. [2004] revealed that truncation effects only became apparent when the sand surface $\left(z_{s}\right)$ was approximately half a capillary fringe height 


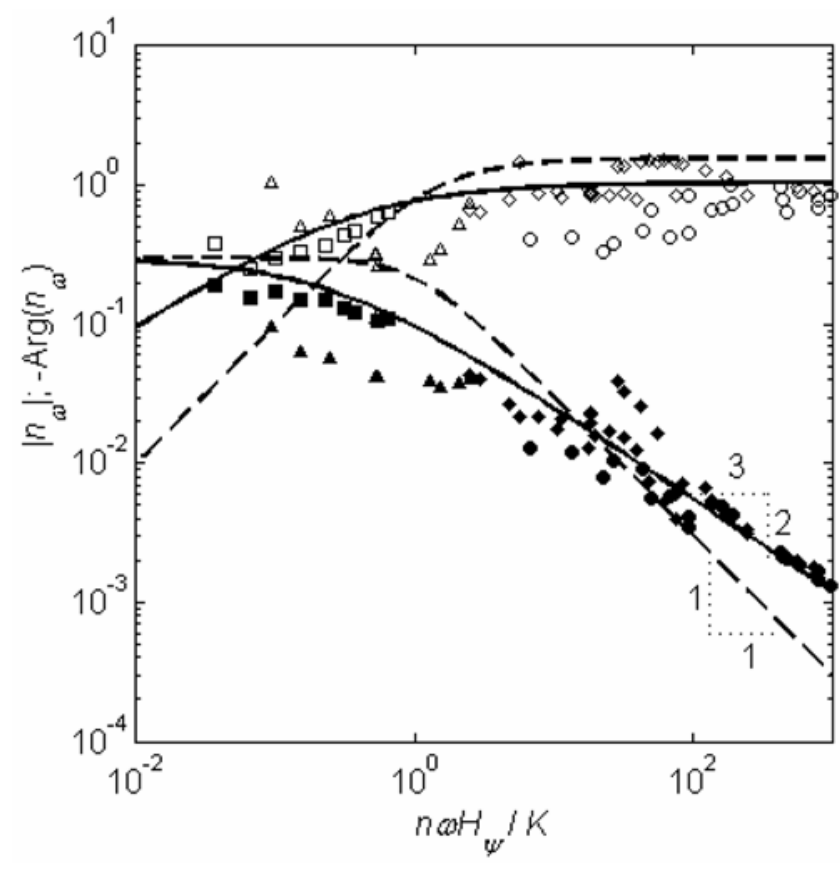

Figure 6. Comparison of the Green and Ampt [1911] complex effective porosity model, equation (7) (dashed lines), with the sand column data and empirical curve fit, equation (5) (solid lines). The data symbols are as defined for Figure 4.

above the maximum water table height $\left(h_{\max }\right)$. That is, truncation effects become important when $z_{s} \leq h_{\max }+$ $0.5 H_{\psi}$.

\section{Comparison With the Green and Ampt [1911] Capillary Fringe Model}

[27] Nielsen and Perrochet [2000] showed that using the Green and Ampt [1911] approximation of the fringe leads to the following linear frequency response function for the water table in the sand column,

$$
F(\omega)=\frac{1+i \frac{n \omega D}{K} \frac{H_{\psi}}{D}}{1+i \frac{n \omega D}{K}\left(1+\frac{H_{\psi}}{D}\right)}
$$

In order to compare this with the present data (i.e., equation (5)) the complex effective porosity model corresponding to the Green and Ampt [1911] approximations is found by inserting (6) into (4) which yields,

$$
n_{\omega}=\frac{n}{1+i \frac{n \omega H_{\psi}}{K}}
$$

The comparison of the Green and Ampt model (equation (7)) with the data (equation (5)) is shown in Figure 6 which highlights two main discrepancies between the two: (1) The Green and Ampt [1911] model (dashed line) predicts $\left|n_{\omega}\right|$ to have an asymptotic slope of -1 whereas the data (solid line) indicate a slope of $-2 / 3$. (2) The asymptotic value of $\operatorname{Arg}\left\{n_{\omega}\right\} \approx-\pi / 2$ for the Green and Ampt model (dashed line) as opposed to $\operatorname{Arg}\left\{n_{\omega}\right\} \approx-\pi / 3$ according to the data (solid line).

[28] In other words, and referring to the definition of $n_{\omega}$ (equation 1), as the high-frequency limit $\left(n \omega H_{\psi} / K \rightarrow \infty\right)$ is approached the Green and Ampt model tends to overpredict both the damping of the total moisture, $\left|n_{\omega}\right|$, and its phase lag, $\operatorname{Arg}\left\{n_{\omega}\right\}$, relative to the corresponding water table quantities. That is, for higher-frequency ocean wind and long-wave forcing of beach groundwater systems, the Green and Ampt model is inadequate.

[29] When applied to obtain a capillarity correction term for the Boussinesq equation the Green and Ampt [1911] type approach yields an extra derivative term of the form $\partial / \partial t\left(\partial^{2} h / \partial x^{2}\right)$ [Parlange and Brutsaert, 1987]. The asymptotic " $2 / 3$ " trend observed in the data however would require a two thirds-order derivative of the form $\partial^{2 / 3} / \partial t^{2 / 3}\left(\partial^{2} h / \partial x^{2}\right)$ as suggested by Nielsen and Turner [2000].

[30] All previous analytical and numerical considerations of capillarity effects on free surface flow have been based on Green and Ampt [1911] type approximations [e.g., Parlange and Brutsaert, 1987; Barry et al., 1996; Li et al., 1997; Jeng et al., 2005] due to its simplicity. The present data however has highlighted significant limitations in this approach, particularly in the high-frequency limit. Limitations of the model when applied to the 2D case of a propagating water table wave have also been identified [Cartwright et al., 2003, 2004]. As such it is suggested that further development of the problem be directed toward improving the understanding of the processes involved in the coupling of the saturated zone with the capillary fringe.

\section{Comparison With Nonhysteretic Numerical Models}

[31] The present database provides an opportunity to evaluate the performance of numerical solutions of the well-known Richards' [1931] equation for partially saturated flow. Here numerical solution is undertaken using the van Genuchten [1980] saturation-pressure model,

$$
S_{e}=\frac{\theta-\theta_{r}}{\theta_{s}-\theta_{r}}=\left[\frac{1}{1+(\alpha \psi)^{\beta}}\right]^{m}
$$

where $S_{e}$ is the effective saturation, $\theta, \theta_{r}$ and $\theta_{s}$ are the local, residual and saturated moisture contents respectively, $\psi$ is the suction pressure head and $\alpha, \beta$ and $m=1-1 / \beta$ are empirical curve fitting parameters. The fit of equation (8) to the measured first drying curves is shown in Figure 3 with the best fit parameters summarized in Table 3 .

[32] The numerical results were subjected to the same analysis described in sections 3 and 3.2 to determine the complex effective porosity over a range of oscillation frequencies. Figure 7 compares the nonhysteretic numerical predictions using the three sets of van Genuchten [1980] parameters summarized in Table 3 and obtained from drying experiments with the sand column data. Like the Green and Ampt [1911] model (see section 5), all three models tend to overpredict both the damping of the total moisture $\left(\left|n_{\omega}\right|\right)$ and its phase lag $\left(\operatorname{Arg}\left\{n_{\omega}\right\}\right)$ relative to the corresponding water table quantities in the high-frequency limit $\left(n \omega H_{\psi} / K\right)$, i.e., 
Table 3. Summary of Parameters Used in the Numerical Simulations ${ }^{\mathrm{a}}$

\begin{tabular}{lcccccc}
\hline $\begin{array}{c}d_{50}, \\
\mathrm{~mm}\end{array}$ & $\begin{array}{c}K_{s}, \\
\mathrm{~m} \mathrm{~s}^{-1}\end{array}$ & $\begin{array}{c}\theta_{s}, \\
\mathrm{~cm}^{3} \mathrm{~cm}^{-3}\end{array}$ & $\begin{array}{c}\theta_{r}, \\
\mathrm{~cm}^{3} \mathrm{~cm}^{-3}\end{array}$ & $\begin{array}{c}\alpha, \\
\mathrm{m}^{-1}\end{array}$ & $\beta$ & $H_{\psi}, \mathrm{m}$ \\
\hline 0.082 & $2.8 \times 10^{-5}$ & 0.38 & 0.06 & 0.68 & 10 & 1.52 \\
0.2 & $4.7 \times 10^{-4}$ & 0.38 & 0.09 & 1.7 & 9 & 0.62 \\
0.78 & $2.5 \times 10^{-3}$ & 0.41 & 0.08 & 11 & 20 & 0.092 \\
\hline
\end{tabular}

${ }^{\mathrm{a}}$ The moisture retention parameters correspond to the first drying curve.

the frequency range corresponding to forcing from oceanic wind and long waves.

\section{A Curious Relationship Between the van Genuchten $\boldsymbol{\beta}$ Parameter and $\boldsymbol{n}_{\boldsymbol{\omega}}$}

[33] During the present numerical experiments it was discovered that a somewhat curious relationship exists between $n_{\omega}$ and the van Genuchten [1980] $\beta$ parameter as shown in Figure 8. It turns out that the asymptotic slope of $\left|n_{\omega} / n\right|$ is given by the negative of van Genuchten's $m(=1-1 / \beta)$ parameter (see equation (8)). Therefore to obtain the $-2 / 3$ slope seen in the data requires that $m=2 / 3$ and hence that $\beta=3$ as shown by the bold dashed curve in Figure 8. Using $\beta=\infty$ would yield the slope -1 , corresponding to the Green and Ampt model (equation (7)) and to the step saturation-pressure model $S_{e}=1$ for $|\psi|<$ $1 / \alpha$ and $S_{e}=0$ for $|\psi|>1 / \alpha$, where $\alpha$ is a shape factor of

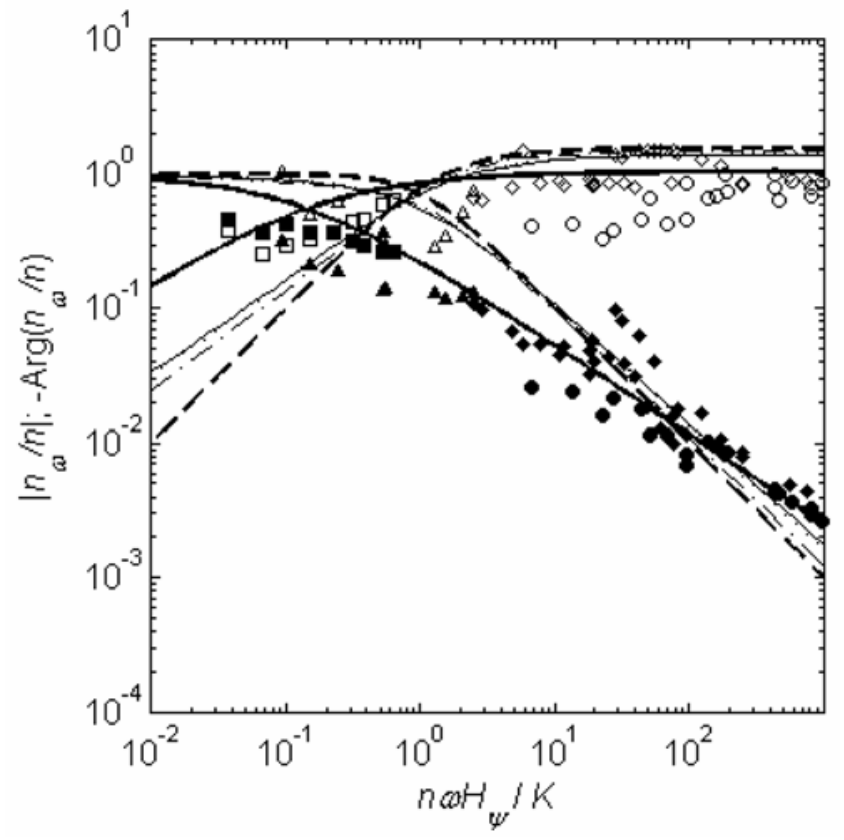

Figure 7. Comparison of simulated complex effective porosities using measured parameters against sand column observations. The thin lines denote the numerical simulations based on the measured parameters for the $d_{50}=0.082$ $\mathrm{mm}$ (dashed), $d_{50}=0.2 \mathrm{~mm}$ (solid) and $d_{50}=0.78 \mathrm{~mm}$ (dash-dotted) sediments. The thick solid and dashed lines denote the empirical and theoretical Green and Ampt models, respectively. The data symbols are as defined for Figure 4.

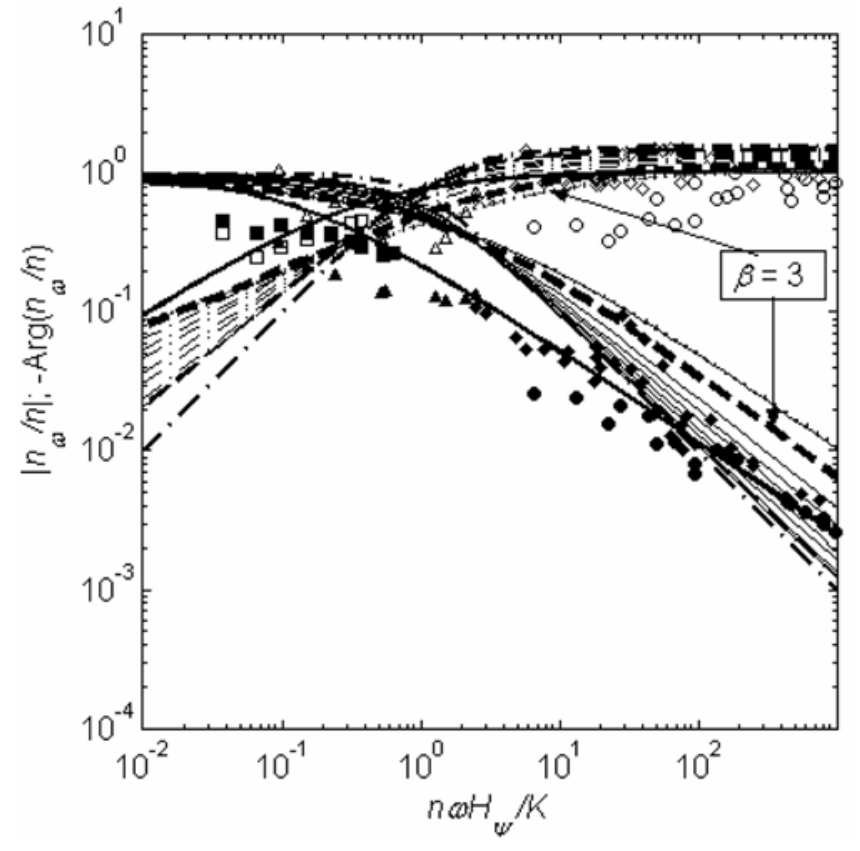

Figure 8. The influence of the van Genuchten parameter $\beta$ on simulated $\left|n_{\omega} / n\right|$ and $-\operatorname{Arg}\left\{n_{\omega} / n\right\}$ for the range $\beta=[2.5$; $3 ; 4 ; 6 ; 8 ; 10 ; 15 ; 20]$ (thin lines). The thick dashed line denotes the $\beta=3$ curves, and the thick solid and dash-dotted lines denote the empirical and theoretical Green and Ampt models, respectively. Parameters used are $D=0.57 \mathrm{~m}, A=$ $0.17 \mathrm{~m}, \alpha=2.3 \mathrm{~m}^{-1}, \theta_{s}=0.385, \theta_{r}=0.065$, and $K_{s}=$ $0.00047 \mathrm{~m} / \mathrm{s}$. The data symbols are as defined for Figure 4 .

the moisture retention curve [e.g., Rooij and Cho, 1999] which approaches the inverse of the air-entry pressure for step-like moisture distributions.

[34] A series of simulations were conducted varying the van Genuchten $[1980] \alpha$ parameter over the range $0.5 \mathrm{~m}^{-1} \leq$ $\alpha \leq 10 \mathrm{~m}^{-1}$ but was seen to have no influence on the inferred values of $n_{\omega}$ over the range of saturations tested.

[35] At this point this finding remains somewhat of a curiosity and the physical implications of which are not presently obvious. However, some measure of appropriateness of adopting a $\beta=3$ moisture retention curve may be determined by comparing it with the measured drying and wetting curves. In the absence of a measured first wetting curve for the present materials, a hysteresis ratio, $\zeta=\alpha^{w} / \alpha^{d}=2$, is adopted to calculate a wetting curve using equation (8). This value of $\zeta$ was suggested by Kool and Parker [1987] who measured the wetting and drying curves for eight different soils and found an average value of $\zeta \approx 2$.

[36] Figure 9 compares the measured first drying curve, calculated wetting curve and a "best fit" $\beta=3$ curve for each of the three materials. Adding to the curiosity of this finding, the $\beta=3$ curve has significantly changed the character of the moisture retention curve to one that is more indicative of a more poorly sorted material. One may argue that because much of the $\beta=3$ curve lies within the bounds of the wetting-drying curve envelope it could be taken to be reasonably representative of the porous medium as some kind of "average" distribution which somehow implicitly accounts for hysteresis scanning loops. However, the fact 
that $\beta=3$ curve lies outside the bounds of the wetting and drying curves at the saturation extremities raises the question of how physical the result may be as the saturated end of the distribution is important for water table oscillations.

[37] Although the discussion above maybe interesting speculation, the question still remains: Is there a physical reason why the $\beta=3$ curve is able to capture dynamics of the hysteretic oscillations? Or is it merely a mathematical coincidence?

[38] The finding is promising in that if it is not just a numerical coincidence then a single "nonhysteretic" moisture retention curve (which does not coincide with the first drying curve) that implicitly accounts for hysteresis effects can be employed as opposed to computationally expensive hysteresis algorithms.

\section{Comparison With a Hysteretic Numerical Model}

[39] It has been shown that consideration of hysteresis in the numerical solution of the Richards [1931] equation greatly improves the performance of many numerical models [e.g., Lehman et al., 1998; Stauffer and Kinzelbach, 2001; Werner and Lockington, 2003]. Werner and Lockington [2003] used the limited amount of sand column data from Nielsen and Perrochet [2000] to validate their model and cited large disagreement between their model predictions and those obtained using the constant complex effective porosity model derived by Nielsen and Perrochet [2000].

[40] Figure 10 compares the hysteretic simulations of Werner and Lockington [2003] with the additional data from the present study and the agreement is seen to be good over the entire range of frequencies $\left(R^{2}=0.69\right.$ for $|F|$ and 0.57 for $\arg \{F\})$. In addition, the present frequencydependent complex effective porosity model (equation (5)) also provides good agreement with the data $\left(R^{2}=0.60\right.$ for
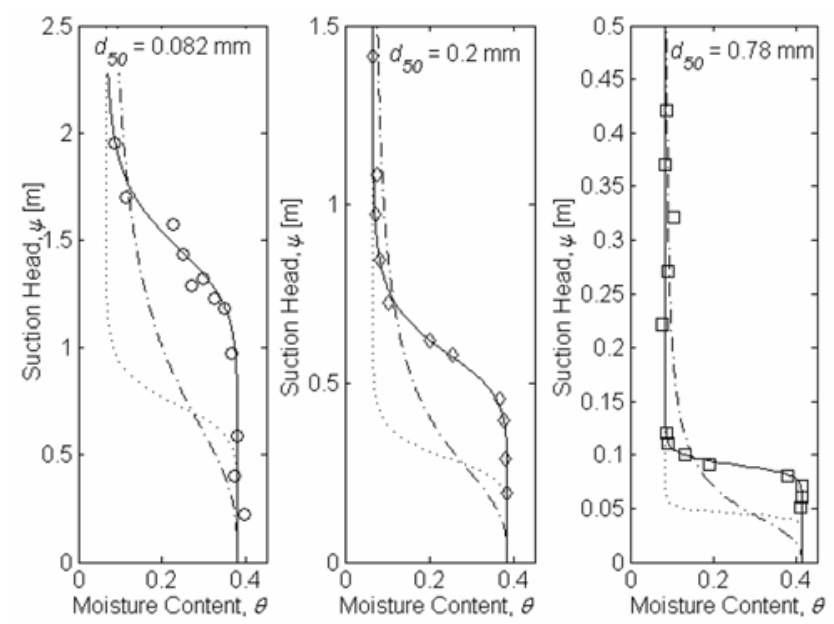

Figure 9. Moisture retention curves for all three sediment types. Symbols denote measured first drying curve data and the lines represent the following van Genuchten curves: the best fit to the measured first drying curve (solid line), the first wetting curve calculated with the hysteresis ratio, $\alpha^{w} / \alpha^{d}=2$ (dashed line), and the curve with $\beta=3$ and $\alpha=\alpha^{w}=2 \alpha^{d}$ (dash-dotted line).
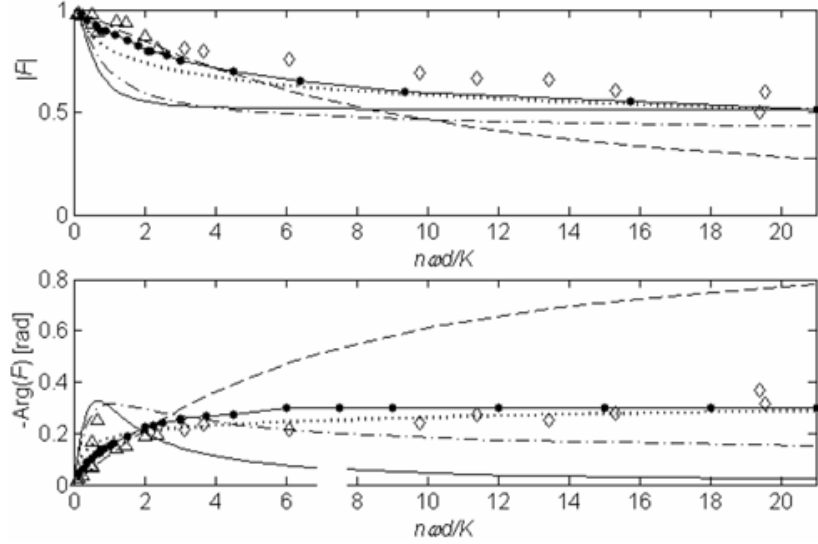

Figure 10. Comparison of the observed frequency response with various model predictions. Symbols denote data from Nielsen and Perrochet [2000] (triangles) and the present study (diamonds). The curves correspond to the hysteretic (solid line with dots) and nonhysteretic (dashdotted line) numerical models of Werner and Lockington [2003] and three complex effective porosity models: the constant $n_{\omega}$ model of Nielsen and Perrochet [2000] (dashed line), the Green and Ampt [1911] $n_{\omega}$ model (solid line), and the present empirical $n_{\omega}$ model (dotted line).

$|F|$ and 0.52 for $\arg \{F\})$, overcoming the large discrepancies found when using the constant complex effective porosity model of Nielsen and Perrochet [2000]. The nonhysteretic numerical and Green and Ampt [1911] type models are both seen to have poor agreement with the observed trends.

\section{Conclusions}

[41] Comprehensive measurements of the water table response to simple harmonic forcing at the base of a sand column have been presented and discussed in terms of the complex effective porosity concept [Nielsen and Perrochet, 2000]. The concept and the data are in qualitative agreement with previous modeling work in that the influence of capillarity increases with the dimensionless parameter $n \omega H_{\psi} / K$ [e.g., Barry et al., 1996; Li et al., 1997].

[42] The data however exposes significant shortcomings of the commonly used Green and Ampt [1911] approximation of the capillary fringe in its ability to describe the influence of capillarity on water table oscillations. As the high-frequency limit is approached $\left(n \omega H_{\psi} / K \rightarrow \infty\right)$, the approximation tends to overpredict both the damping $\left(\left|n_{\omega}\right|\right)$ and phase lag $\left(\operatorname{Arg}\left\{n_{\omega}\right\}\right)$ of oscillations in the total moisture relative to those in the water table. This has implications for the modeling of the beach groundwater response to high-frequency wind and long-wave forcing.

[43] To overcome the limitations in the Green and Ampt [1911] approximation an empirical complex effective porosity model has been provided which can be easily applied to existing linear dispersion relations [e.g., Todd, 1960; Nielsen et al., 1997] to correct them for the influence of capillarity.

[44] The simple empirical complex effective porosity model equation (5) has been shown to perform similarly well when compared to the data and the performance of the 
more computationally expensive numerical hysteretic model of Werner and Lockington [2003] while the nonhysteretic and Green and Ampt [1911] models performed poorly.

[45] It is duly noted that the applicability of the present findings from experiments using well-sorted materials may have a limited direct applicability to more poorly sorted materials. However, for the present motivating case of sandy beach groundwater systems, the findings provide a sound basis for more accurately quantifying the dynamic coupling of the ocean-aquifer system or other well-sorted aquifers. The findings may also provide a useful starting point for an investigation into the behavior of water table oscillations in poorly sorted soils under the influence of capillarity.

\section{Notation}

$\alpha$ van Genuchten parameter, $\mathrm{m}^{-1}$.

$\alpha^{d} \quad$ van Genuchten parameter (drying), $\mathrm{m}^{-1}$.

$\alpha^{w}$ van Genuchten parameter (wetting), $\mathrm{m}^{-\mathrm{i}}$.

$\beta$ van Genuchten parameter.

$D$ mean aquifer thickness, $\mathrm{m}$.

$d_{50}$ median grain diameter, $\mathrm{mm}$.

$\eta \quad$ water table elevation, $\mathrm{m}$.

$\eta_{0}$ driving head elevation, $m$.

$F$ frequency response function.

$|F|$ amplitude ratio between water table and driving head, $\mathrm{m}$.

$\phi$ phase lag between water table and driving head, rad.

$g$ acceleration due to gravity, $\mathrm{m} \mathrm{s}^{-2}$.

$h$ water table elevation $(=D+\eta), \mathrm{m}$.

$h_{\max }$ maximum water table elevation, $\mathrm{m}$.

$h^{*}$ piezometric head, m.

$h_{0}$ driving head elevation $\left(=D+\eta_{0}\right), \mathrm{m}$.

$h_{\text {tot }}$ equivalent saturated height of total moisture, $\mathrm{m}$.

$H_{\psi}$ equivalent saturated steady capillary fringe height, $\mathrm{m}$.

$K$ saturated hydraulic conductivity, $\mathrm{m} \mathrm{s}^{-1}$.

$m$ van Genuchten parameter.

$n$ drainable porosity.

$n_{d}$ complex effective porosity notation used by Nielsen and Perrochet [2000] $\left(=n_{\omega}\right)$.

$n_{\omega}$ complex effective porosity.

$\theta$ local moisture content.

$\theta_{s}$ saturated moisture content.

$\theta_{r}$ residual moisture content.

$\rho$ density, $\mathrm{kg} \mathrm{m}^{-3}$.

$S_{e}$ effective saturation.

$T$ oscillation period, $\mathrm{s}$.

$t$ time, s.

$\omega$ angular frequency, $\operatorname{rad~} \mathrm{s}^{-1}$.

$x$ horizontal coordinate, $\mathrm{m}$.

$\psi$ suction head, $\mathrm{m}$.

$z_{0}$ piezometer elevation within the saturated zone, $\mathrm{m}$.

$z_{s}$ sand surface elevation, $\mathrm{m}$.

$\zeta$ hysteresis ratio.

[46] Acknowledgments. The present research work has been supported in part by the Australian Research Council (ARC) as project number DP0346461 and by the Collaborative Research Centre (CRC) for Sustainable Tourism as project number 52001. The financial assistance of an Australian Postgraduate Award to the first author is also gratefully acknowledged.

\section{References}

Barry, D. A., S. J. Barry, and J.-Y. Parlange (1996), Capillarity correction to periodic solutions of the shallow flow approximation, in Mixing in Estuaries and Coastal Seas, Coastal Estuarine Stud., vol. 50, edited by C. B. Pattiaratchi, pp. 496-510, AGU, Washington D. C.

Cartwright, N., P. Nielsen, and S. L. Dunn (2003), Water table waves in an unconfined aquifer: Experiments and modeling, Water Resour. Res., 39(12), 1330, doi:10.1029/2003WR002185.

Cartwright, N., P. Nielsen, and L. Li (2004), Experimental observations of watertable waves in an unconfined aquifer with a sloping boundary, $A d v$. Water Resour., 27, 991-1004.

Duke, H. R. (1972), Capillary properties of soils-Influence upon specific yield, Trans. ASAE, 15, 688-691.

Elfrink, B., and T. E. Baldock (2002), Hydrodynamics and sediment transport in the swash zone: A review and perspectives, Coastal Eng., 45(3), $149-167$.

Gillham, R. W. (1984), The capillary fringe and its effect on water-table response, J. Hydrol., 67, 307-324.

Green, W. H., and G. A. Ampt (1911), Studies on soil physics 1. The flow of air and water through soils, J. Agric. Sci., 4(1), 1-24.

Jeng, D. S., B. R. Seymour, D. A. Barry, L. Li, and J.-Y. Parlange (2005), New approximation for free surface flow of groundwater: Capillarity correction, Adv. Water Resour., in press.

Kool, J. B., and J. C. Parker (1987), Development and evaluation of closedform expressions for hysteretic soil hydraulic properties, Water Resour. Res., 23(1), 105-114.

Lehman, P., F. Stauffer, C. Hinz, O. Dury, and H. Flühler (1998), Effect of hysteresis on water flow in a sand column with a fluctuating capillary fringe, J. Contam. Hydrol., 33(1-2), 81-100.

Li, L., D. A. Barry, J.-Y. Parlange, and C. B. Pattiaratchi (1997), Beach water table fluctuations due to wave run-up: Capillarity effects, Water Resour. Res., 33(5), 935-945.

Li, L., D. A. Barry, F. Stagnitti, and J.-Y. Parlange (2000), Groundwater waves in a coastal aquifer: A new governing equation including vertical effects and capillarity, Water Resour. Res., 36(2), 411-420.

Nachabe, M. H. (2002), Analytical expressions for transient specific yield and shallow water table drainage, Water Resour. Res., 38(10), 1193, doi:10.1029/2001WR001071.

Nielsen, P., and P. Perrochet (2000), Watertable dynamics under capillary fringes: Experiments and modelling, Adv. Water Resour, 23(1), 503515. (Errata, Adv. Water Resour., 23, 907-908, 2000.)

Nielsen, P., and I. Turner (2000), Groundwater waves and water exchange in beaches, paper presented at 27th International Conference on Coastal Engineering, Am. Soc. of Civ. Eng., Sydney, Australia.

Nielsen, P., A. M. Aseervatham, J. D. Fenton, and P. Perrochet (1997), Groundwater waves in aquifers of intermediate depths, Adv. Water Resour., 20, 37-43.

Parlange, J.-Y., and W. Brutsaert (1987), A capillarity correction for free surface flow of groundwater, Water Resour. Res., 23(5), 805-808.

Richards, L. A. (1931), Capillary conduction of liquids through porous medium, Physics, 1, 318-333.

Rooij, G. H., and H. Cho (1999), Modelling solute leaching during fingered flow by integrating and expanding various theoretical and empirical concepts, Hydrol. Sci. J., 44(3), 447-465.

Stauffer, F., and W. Kinzelbach (2001), Cyclic hysteretic flow in porous medium column: Model, experiment, and simulations, J. Hydrol., $240(3-4), 264-275$.

Todd, D. K. (1960), Ground Water Hydrology, 2nd ed., 336 pp., John Wiley, Hoboken, N. J.

van Genuchten, M. T. (1980), A closed form equation for predicting the hydraulic conductivity of unsaturated soils, Soil Sci. Soc. Am. J., 44, $892-898$.

Werner, A. D., and D. A. Lockington (2003), Influence of hysteresis on tidal capillary fringe dynamics in a well-sorted sand, Adv. Water Resour., 26, 1199-1204.

N. Cartwright, School of Engineering, Griffith University, PMB50 Gold Coast Mail Centre, Gold Coast, Qld 9726, Australia. (n.cartwright@griffith. edu.au)

P. Nielsen, Division of Civil Engineering, University of Queensland, St. Lucia, Qld 4072, Australia.

P. Perrochet, Centre of Hydrogeology, University of Neuchâtel, avenue du 1er-Mars 26, CH-2000 Neuchâtel, Switzerland. 